\title{
Des effets du divorce et du non-divorce sur les enfants
}

\author{
Claude Martin $\quad$ Sociologue, directeur de recherche au CNRS. Centre de \\ recherche sur I'action politique en Europe/IEP de Rennes.
}

C et article propose de revenir sur les principales conclusions des travaux de recherche existant au plan international et d'explorer la question des effets du non-divorce pour les couples qui connaissent le désamour et le conflit conjugal. Qu'en estil en somme de ces couples qui renoncent au divorce pour épargner leurs enfants, au risque de produire des effets tout aussi dévastateurs voire plus dévastateurs encore s'ils s'installent dans la conflictualité quotidienne, de ces couples qui décident de vivre ensemble séparés - mode de vie qualifié de «living together apart»?

La question du divorce et de ses effets sur les enfants est posée de manière récurrente depuis le siècle des Lumières. S'il est admis généralement que le divorce ou la séparation des parents constituent une épreuve douloureuse pour les enfants, il est plus difficile de tirer des conclusions définitives sur le lien exact entre cette expérience et le fait d'éprouver durablement des difficultés psychologiques, sociales, scolaires, ou comportementales. L'expérience du divorce est, en effet, d'une grande complexité : elle peut inaugurer des trajectoires variées, provoquer des cassures et des préjudices graves, comme fournir des exemples de résilience (1). Il est donc difficile d'isoler ce phénomène du faisceau des variables en cause [variables socio-économiques et culturelles, variables relationnelles, rôle de l'État-providence (2), notamment]. Tout aussi complexe est l'évaluation de ses effets sur la moyenne ou la longue durée. Si l'immédiat après-divorce est souvent marqué par d'importantes perturbations pour les enfants (liées en particulier à la réorganisation du rythme et des conditions de vie et d'habitat, aux tensions persistantes entre les parents, au problème de conflit de loyauté, à l'appauvrissement du ménage, etc.), le temps long est susceptible de permettre une stabilisation et un apaisement des relations familiales.
L'instabilité des couples concerne aujourd'hui tous les milieux sociaux, différentes générations, différentes «cultures » ou sphères sociales, et ce dans la plupart des pays développés. Son impact varie considérablement selon les valeurs et les normes en vigueur. Ainsi, manifestement, une partie des difficultés rencontrées par les adultes et les enfants concernés est liée à la désapprobation sociale et aux différentes formes de stigmatisation dont ils font I'objet. Quand une très large majorité des ménages était en France composée de couples mariés avec des enfants légitimes, avec des taux de divortialité inférieurs à $10 \%$ et une norme familiale dominante (la "famille bourgeoise »), la question du divorce et de ses effets se posait dans des termes bien différents de ceux que l'on connaît aujourd'hui où, pour cent mariages une année donnée, on enregistre quarante-sept divorces (Vanderschelden, 2006).

Malgré cette diversité de trajectoires et de facteurs en cause, nombre de travaux ont tenté et tentent encore régulièrement d'établir des causalités entre divorce et bien-être (ou plutôt mal-être) des enfants. C'était ainsi manifestement le cas au début du XX $X^{\mathrm{e}}$ siècle. Des criminologues et pédopsychiatres de cette époque montraient ou croyaient démontrer que ces effets étaient toujours négatifs et que les enfants ayant subi le divorce ou la séparation de leurs parents éprouvaient toujours plus de difficultés que les enfants demeurant dans des familles biparentales simples, unissant leurs deux géniteurs. Ce diagnostic est en partie lié aux méthodes utilisées et aux effets de sélection de ces enquêtes.

Il faudra attendre les années 1970 et 1980 pour que des psychologues et des sociologues questionnent ce paradigme dominant et (ré)introduisent la controverse, en défendant que le 
divorce, en tant que mode de résolution de crise, est susceptible d'avoir des effets moins négatifs que le fait d'être élevé dans des familles où règne un conflit permanent sans séparation. Dans cette perspective où le divorce est conçu comme un moindre mal, la comparaison doit moins porter sur les écarts de bien-être repérables entre les enfants de familles dites "intactes» et les enfants de familles dissociés, mais entre les enfants qui connaissent un contexte familial marqué par le conflit conjugal, avec ou sans séparation. La variable principale dont on évalue les effets n'est plus, dès lors, le divorce, mais le conflit.

Cet article propose de revenir sur les principales conclusions des travaux de recherche existant au plan international, mais également $d^{\prime}$ interroger la faiblesse des sciences sociales face aux idées reçues et aux besoins de simplification que manifeste régulièrement l'opinion publique (Martin, 2003 b). On peut ainsi se demander si l'audience d'un certain nombre d'interprétations savantes n'est pas proportionnelle à leur conformité à une représentation dominante, à leur résonance dans le débat public, au risque de faire fi d'autres résultats acquis au plan de la recherche. Et, au-delà, on peut se demander si la focalisation sur les couples dissociés ne masque pas un autre phénomène, bien peu étudié par les sociologues, comme les effets du non-divorce pour les couples qui connaissent le désamour et le conflit conjugal. Cette terra incognita est ici explorée : qu'en est-il en somme de ces couples qui renoncent au divorce pour épargner leurs enfants, au risque de produire des effets tout aussi dévastateurs, voire plus dévastateurs encore, s'ils s'installent dans la conflictualité quotidienne? Si l'important pour le bien-être des enfants, comme semblent le démontrer les connaissances acquises, est moins la séparation, la rupture comme telle, que le conflit et la manière dont se déroule une rupture en amont et en aval, il serait urgent de développer les connaissances sur "I'avant-divorce », en particulier sur les couples qui demeurent des parents alors qu'ils ne sont plus un couple, tout en décidant de ne pas se séparer - mode de vie que l'on qualifie de "living together apart » (3), c'est-àdire des couples féconds qui, malgré une profonde insatisfaction conjugale et des désaccords persistants, choisissent de rester ensemble pour éviter à la fois de confronter leurs enfants à la séparation, mais aussi à ses conséquences économiques et sociales.

\section{Penser le divorce : des idées récurrentes depuis plus de trois siècles}

L'histoire des idées sur le divorce montre qu'une partie des questions essentielles qui organisent aujourd'hui encore les débats et les réformes ont été posées il y a plus de trois siècles. Autrement dit, les débats contemporains - qui fonctionnent pour une bonne part à l'amnésie - témoignent des chocs idéologiques permanents que soulèvent ces comportements conjugaux. Comme le disait fort bien le doyen Jean Carbonnier, on peut penser que se perpétue ainsi "l'archétype de l'indissolubilité » auquel il serait si difficile de renoncer (Carbonnier, 1995, p. 167). Ainsi, la notion de "divorce-faillite» ou de "divorce pour incompatibilité d'humeur» - qui a donné lieu à la plus récente réforme du divorce en 2005 (4) - est loin d'être une nouveauté. Avant même que ne s'engage le débat des Encyclopédistes sur la question du divorce, qui créera les conditions de possibilité de son adoption par les révolutionnaires en 1792 sur un mode particulièrement libéral, avait déjà eu lieu, près de cent cinquante ans auparavant en Angleterre, une autre révolution: le puritanisme. Dans le texte qu'il publie pour la première fois en 1643, John Milton (2005) soutient que la meilleure défense du mariage réside précisément dans la possibilité du divorce, car « penser le mariage suppose de penser le divorce, d'abord dans la déliaison la moins malheureuse possible de deux libertés égales qui cherchent leur bonne distance... La fragilité du lien est alors paradoxalement sa force, car il ne tient que du consentement sans cesse renouvelé, de la possibilité de le rompre »(Abel et Laugier, 2005, p. 326). La lecture que propose J. Milton des premiers versets de la Genèse le conduit à réfuter I'ordonnance d'indissolubilité appliquée par l'Église catholique romaine, dont il dénonce l'hypocrisie:

"Bien que Dieu, lors de l'institution du mariage, nous enseigne pour quelle fin il le fit, en des termes impliquant expressément la conversation heureuse et joyeuse entre l'homme et la femme afin de réconforter l'homme et de le remettre de I'affliction d'une vie solitaire, en ne mentionnant le but de la procréation que par la suite, comme une fin secondaire (...), pourtant, si maintenant un couple est passé une fois dans les mains de l'Eglise et qu'il a goûté tant soit peu au lit nuptial, s'il s'avère un jour que les deux conjoints se sont trompés sur leurs dispositions respectives parce qu'ils se sont mépris sur la personne, parce qu'ils se sont

(3) En référence à l'expression « living apart together » qui entend désigner les couples non cohabitants (Le Gall et Martin, 1988).

(4) La réforme du divorce de janvier 2005 a eu pour objectif de " dédramatiser » les ruptures, d'apaiser les démarches judiciaires, mais aussi de modifier les conditions du "divorce-faillite » en réduisant de six à deux ans l'absence de vie commune avant l'assignation en divorce. On reprend ici quelques éléments d'un texte déjà publié dans le dictionnaire des sciences humaines (Martin, 2006). 
cachés la vérité ou parce qu'ils sont mal tombés s'il s'avère qu'à cause de leur différence de tempéraments, de caractères ou de constitutions, (...) ils ne peuvent vivre en harmonie ou vivre heureux tous les jours de leur vie, cependant il leur faudra, ... malgré l'aversion qu'ils ont l'un pour l'autre, rester vaille que vaille unis dans une lassitude sans nom et désespérer de toute joie de vivre ensemble dans I'ordonnance que Dieu a prévue précisément à cet effet. Quelle calamité est-ce là ! (...) Tout cela, nous ne pouvons l'attribuer à juste titre qu'à un seul auteur : au droit canon et à ses adhérents...» (Milton, 2005, pp. 81-82).

Scellé définitivement sans tenir compte des conditions du bonheur, un tel mariage n'a pour J. Milton aucune légitimité. Comme le souligne Irène Théry " délier ces deux-là qui n'étaient pas faits l'un pour I'autre, n'est pas aller contre l'Écriture, car ceux qui tôt ou tard découvrent à quel point ils sont malheureux ensemble, n'ont jamais été unis, ni réellement mariés» (Théry, 2004, p. 77). Si la finalité du texte de J. Milton est principalement de s'attaquer à la "superstition papiste ", il définit aussi une autre conception du mariage, une conception privée, intime, individualisée, de ce lien social, une conception qui met au centre la recherche du bonheur, la " conversation conjugale ", $\mathrm{au}$ sens d'un "pur plaisir de passer du temps ensemble »(Cavell, 1993).

Cette légitimation du divorce comme déliaison de ce qui n'aurait pas dû être uni butte néanmoins sur un certain nombre d'écueils qui ouvriront, jusqu'à aujourd'hui, débats et recherches de compromis. Le premier écueil est l'inégalité de posture des protagonistes dans ce lien social particulier et la différence fondamentale entre celui ou celle qui veut se libérer de ce lien et celui ou celle qui subit cette demande. Un autre écueil réside dans la place faite aux enfants et aux conséquences pour eux de cette rupture. Nombre d'auteurs tenteront d'imaginer des solutions. Le droit et la régulation judiciaire sont supposés répondre au premier écueil. Ainsi, Pierre-Joseph Proudhon qui, au XIX ${ }^{\mathrm{e}}$ siècle, ne voulait pas démordre de l'indissolubilité tout en ayant conscience de l'inéluctabilité de l'usure conjugale, "fut acculé à inventer un remède juridique plus audacieux encore que le divorce: les conjoints disjoints ne seraient pas autorisés à contracter de nouveaux mariages, mais ils auraient licence de tempérer leurs solitudes par des concubinages licites. Et de mettre sur pied un statut du concubinat légal, comme d'une union de second rang" (Carbonnier, 1988, p. 233). Quant au second écueil, il inspirera d'autres types de compromis, dont celui imaginé au siècle des Lumières par Christian Wolff (1679-1754), consistant à autoriser le consentement mutuel en l'absence d'enfant ou quand l'éducation de ceux-ci est achevée et, dans le cas contraire, à interdire le divorce.

\section{Les effets du divorce sur les enfants : éléments pour un bilan}

Du début du XXe siècle à aujourd'hui, les conséquences psychologiques et sociales de l'instabilité familiale sur les enfants ont généralement été appréciées en termes négatifs (Martin, 1997). L'idée reçue sur le sujet consiste à penser que les " enfants du divorce» (5) sont confrontés à de nombreuses difficultés, allant de la dépression, aux comportements agressifs, de la délinquance à l'échec scolaire, etc., et que le divorce est la principale cause de ces difficultés : la variable indépendante. Criminologues et psychiatres ont joué un rôle central dans la construction de ce point de vue à la fin du XIXe et au début du XX $X^{\mathrm{e}}$ siècle, en particulier en tentant d'établir une causalité entre instabilité familiale et délinquance (Heuyer, 1952) (6). Cette lecture a eu au moins pour conséquences, d'une part, de renforcer l'idée que la famille nucléaire et légitime était une des principales clés de voûte de la société, et donc que la fragilisation de cette " cellule de base " pouvait menacer la paix sociale (7) et, d'autre part, de construire une causalité simple entre un problème aussi complexe que la délinquance et un facteur isolé $d^{\prime} u n$ faisceau d'autres facteurs: la structure familiale (Lefaucheur, 1996).

Ces travaux antérieurs ont eu un impact non négligeable sur des générations d'éducateurs, d'enseignants, de professionnels de la famille et de I'enfance, en leur fournissant un modèle simple et apparemment efficace pour expliquer nombre de problèmes sociaux (incivilité, délinquance, violence, échec scolaire, toxicomanie, déséquilibre psychologique et émotionnel, etc.). Pour autant, au cours des trente ou quarante dernières années, les sciences sociales - et cette fois plutôt la sociologie, la démographie, voire la science politique - sont parvenues à montrer que séparation et divorce représentaient une expérience sociale complexe, dont les conséquences ne sont

\footnotetext{
(5) Curieuse catégorie qui ne désigne pas même ce qu'elle prétend désigner, puisque ces enfants ne sont pas issus du divorce mais ont connu le divorce de leurs parents. Ce faisant, cette expression a cependant un effet : celui de donner à penser que tous ces enfants partagent une expérience sociale fondamentale qui les distingue de tous les autres enfants : avoir connu la séparation et le divorce de leurs parents.

(6) Pour une présentation de cette construction savante des causes et des effets du divorce, on se reportera aux travaux de Nadine Lefaucheur (1989) et de Claude Martin (1997).

(7) Comme l'énonçait Léon Gambetta : " II n’y a pas de question sociale, il n'y a que des problèmes familiaux ».
} 
pas aussi mécaniques que I'on avait coutume de les considérer. En nuançant le diagnostic et en montrant le rôle d'autres variables jusque-là quelque peu négligées, ces nouveaux résultats sont ainsi parvenus à réduire le poids et les stigmates qui pesaient sur ces situations familiales. La cause qui semblait entendue : I'instabilité des couples brise les enfants et font d'eux des enfants à risques, était ainsi réexaminée. L'équation se complexifiait et la diversité des variables pertinentes permettant de prendre la mesure de la variété des configurations et des trajectoires post-divorce s'imposait.

Paul R. Amato, un des auteurs de référence sur ces questions aux États-Unis, propose dans un article de 1994 un bilan des travaux anglo-saxons réalisés à l'époque sur les effets à long terme du divorce sur les enfants (Amato, 1994). Il souligne les nombreuses limites méthodologiques des enquêtes disponibles, qu'il s'agisse des méthodes de comparaisons transversales (cross-sectional) ou longitudinales. Le constat semble clair de prime abord: les enfants ayant connu le divorce de leurs parents éprouvent tendanciellement plus de problèmes psychologiques et sociaux, manifestent plus de symptômes et de difficultés psychologiques, ont de moins bonnes performances scolaires, une moins bonne image d'eux-mêmes que les enfants élevés dans des familles biparentales «intactes». Cependant, l'auteur insiste sur les difficultés d'interprétation que soulève ce constat global. En effet, comme il l'écrit lui-même: "Cependant, les différences entre le groupe des enfants issus de familles divorcées ou intactes sont faibles, avec une diversité considérable de réactions des enfants aux divorces, qui dépendent de plusieurs facteurs, comme la quantité et la qualité des contacts avec le parent non résident, les compétences parentales et l'adaptation psychologique du parent gardien, le niveau de conflit entre les parents avant et après le divorce, le degré des difficultés économiques et le nombre des événements stressants qui ont accompagné ou suivi le divorce »(Amato, 1994, p. 143).

Pour sa part, Andrew Cherlin, I'un des plus éminents représentants de la sociologie de la famille aux États-Unis, fait état dans son manuel, publié en 1999, de l'une des recherches qu'il a menées sur le sujet auprès de milliers d'enfants anglais et américains suivis de l'âge de 7 ans à l'âge de 11 ans: "Nous avons découvert que les enfants dont les parents allaient plus tard divorcer montraient déjà plus de problèmes de comportements et de difficultés scolaires que les enfants dont les parents allaient rester ensemble. Ces résultats suggèrent que certains des traumatismes du divorce commencent bien avant la séparation, quand des parents malheureux se séparent. De plus, ils suggèrent que les problèmes que manifestent les enfants après un divorce auraient pu tout aussi bien avoir lieu dans une certaine mesure si les parents en conflit étaient restés ensemble»(Cherlin, 1999, p. 391).

Le ministère de la Justice canadien a également réalisé un état des savoirs sur le sujet en 1997. Ce rapport insiste sur les obstacles méthodologiques et sur les précautions qu'il est nécessaire de prendre avec les conclusions des recherches disponibles, et suggère de privilégier l'analyse des facteurs qui permettent d'expliquer les écarts de ces impacts (le sexe et l'âge de l'enfant au moment du divorce, la situation socioéconomique du ménage, les compétences parentales, le temps écoulé depuis la rupture, les conflits, les réseaux de soutien, la procédure judiciaire, les modalités de garde des enfants, le remariage et les changements de milieu). Le constat est une fois encore nuancé et prudent : "En raison des limites susmentionnées, il est difficile de tirer des conclusions définitives à partir des travaux de recherche publiés " (ministère de la Justice Canada, 1997, p. 5) (8).

\section{" La liaison entre famille dissociée et délinquance est faible..."}

En France, en 1999, Laurent Mucchielli a dressé un bilan de la littérature scientifique francophone et anglophone sur le thème "familles et délinquances ». Le rapport publié en 2000 a donné lieu à plusieurs publications la même année, dont un article intitulé La dissociation familiale favorise-telle la délinquance? Arguments pour une réfutation empirique (9). À I'appui de cette très abondante littérature française, anglaise, américaine et canadienne, I'auteur montre que cette causalité ne joue pas et que la dissociation familiale n'est pas en soi un facteur majeur de comportements déviants : "La liaison entre famille dissociée et délinquance est faible ou nulle pour les délits graves (vols, comportements violents), un peu plus forte pour la consommation de drogues (surtout douces) et surtout significatives pour les comportements problématiques (fugues, absentéisme scolaire, problème de discipline en classe) » (Mucchielli, 2000 b, p. 43). Si les résultats convergent

(8) Les pédiatres canadiens ont également contribué à cette discussion. En 2000, le Comité de pédiatrie psychosociale de la Société canadienne de pédiatrie a publié un article dont l'objectif est explicitement " d'aider les enfants à affronter la séparation de leurs parents» et ainsi d'orienter les interventions et les méthodes des médecins (Comité de pédiatrie psychosociale, 2000). Selon cet article «les recherches laissent supposer que c'est le conflit parental, et non la séparation, qui nuit le plus aux enfants» (p. 238).

(9) Ce diagnostic reprend sur bien des points ce qu'écrivait déjà N. Lefaucheur à la fin des années 1980, voir notamment son rapport «Dissociation familiale et délinquance juvénile ou la trompeuse éloquence des chiffres » (Lefaucheur, 1996). 
sur un point, $c^{\prime}$ est celui qui consiste à dire que le divorce ou la séparation ne provoquent pas de manière mécanique ce genre de passages à l'acte et que ce qui joue renvoie plutôt à la manière dont se déroulent ces événements, en fonction de nombreuses variables, comme le niveau socio-économique et culturel des ménages concernés, les ressources relationnelles mobilisables, les contacts maintenus ou non entre les ex-conjoints, le rôle des grands-parents et des amis, etc. : "La part que la famille prend dans la fabrique de la délinquance doit être recherchée et comprise en des termes de dynamiques relationnelles et de contexte socioéconomique» (ibid, p. 47) (10).

Un autre facteur évoqué par ces travaux de recherche sur les rapports entre familles et délinquance renvoie à l'exercice du contrôle parental, exercice bien souvent tributaire de l'environnement socioéconomique des familles. Les Anglo-Saxons parlent de "supervision des parents» pour désigner le contrôle formel ou informel que les parents exercent sur les sorties de leurs enfants, sur leurs fréquentations, sur leur travail à l'école ou sur leurs activités de loisirs. Cette attitude ou compétence parentale semble directement liée au bien-être personnel des parents et, inversement, son défaut est lié directement aux handicaps économiques et sociaux des parents (chômage, pauvreté). Ainsi, il est d'autant plus difficile d'exercer ce travail de supervision parentale si l'on est soi-même dans une position disqualifiée. L'autorité d'un père serait, par exemple, souvent corrélée avec son insertion sociale et professionnelle, de même que sa situation de dépendance économique et sociale pourrait jouer comme un obstacle dans le processus d'identification du fils et pourrait pousser le père à osciller entre deux positions extrêmes et également inadéquates : le retrait ou l'autoritarisme.

Ce constat rejoint les résultats des recherches de Sébastian Roché et Vincent Tournier sur la délinquance autodéclarée à partir d'une enquête menée auprès de deux mille jeunes âgés de 13 ans à 19 ans. Partant du résultat suivant: "les actes déclarés ne sont pas plus fréquents lorsque les parents sont séparés que dans l'ensemble de l'échantillon », les auteurs insistent sur la question de la veille ou de la vigilance parentale. Cette veille parentale jouerait un rôle central dans la réduction du passage à l'acte incivil ou délictueux et serait même susceptible de compenser le conflit parental pour éviter ce passage. "Si l'on compare une veille forte et une mauvaise entente (les parents veillent, quitte à être en conflit), d'une part, à une veille faible et une bonne entente (les parents se tiennent en retrait, le climat familial est apaisé), d'autre part, on remarque que la première situation est plus favorable que la seconde... Autrement dit, la bonne entente ne peut pas remplacer la veille des parents » (Roché, 2000) (11).

En somme, au regard des connaissances disponibles, "les facteurs socio-économiques s'avèrent bel et bien les facteurs les plus déterminants dans la fabrique de la délinquance, mais de façon indirecte, en ruinant les capacités de contrôle des parentset surtout des pères » (Mucchielli, 2000 c, p. 141) (12). Mais ce n'est pas ce qui est retenu par le discours commun qui confond, par méconnaissance de la vie de ces ménages défavorisés, crainte, honte, soumission, appréhension avec négligence ou démission des parents : "En fait de démission il faut donc se demander si certains parents ont encore la possibilité d'exercer un contrôle adéquat tant leur existence est difficile» (ibid., p. 142) (13). On perçoit ici la principale difficulté de ces résultats. La réponse est nuancée. Elle suppose d'approfondir l'examen, de faire jouer une pluralité de variables et de tenir particulièrement compte des inégalités de trajectoires post-divorce. Mais, ce faisant, elle semble trop complexe pour être reçue par une opinion publique soucieuse de réponses simples à des questions simples.

\section{L'impact du divorce sur la scolarité des enfants : des résultats contrastés}

L'impact du divorce sur la scolarité des enfants est un autre sujet sur lequel a été mobilisée une masse impressionnante de recherches au cours des dernières décennies, à la mesure sans doute de l'inquiétude que suscite aujourd'hui la réussite

\footnotetext{
(10) Nos travaux ont abouti à cette même conclusion à l'aune d'une enquête menée au début des années 1990 sur une cohorte de divorcés et séparés (Martin, 1997).

(11) Voir sur le site http://web.upmf-grenoble.fr/cerat/Recherche/PagesPerso/Roche.html.

(12) Dans son bilan, Laurent Mucchielli insiste également sur d'autres variables qui ont trait à la représentation qu'ont les jeunes des quartiers défavorisés de leur destin social, à l'idée qu'ils se font de leur place dans la société et aussi à ce qu'ils perçoivent de la place qui leur est faite par les institutions et par la société globale. Il évoque le phénomène d' « inversion du stigmate » qui, d'imposé, devient assumé et pousse les jeunes de ces quartiers à se replier sur des comportements délinquants, souvent assumés à l'échelle du groupe de pairs. Pour un bilan, voir aussi Mucchielli (2001) et Aubusson de Carvalay (2002).

(13) Éric Debardieux (2002), auteur d'une vaste enquête sur les mécanismes de la violence des mineurs, dresse le même type de constat lorsqu'il écrit: "Les parents de délinquants que nous avons rencontrés ne sont pas démissionnaires. I/s ne savent plus quoi faire - ce qui n'est pas la même chose - et se trouvent démunis. Cette impuissance est la même que celle de certains professionnels, enseignants, travailleurs sociaux ou policiers. Cette impuissance est bien collective et ne doit pas conduire à culpabiliser les seuls parents» (interview publiée dans le journal Le Monde du 21 mars 2002). Pour retracer la construction médiatique des enjeux de la parentalité, voir Martin (2003 a).
} 
scolaire des enfants. Une fois encore les résultats sont contrastés, mais il est remarquable que certains d'entre eux trouvent à certains moments une véritable audience. Lorsqu'une opinion s'impose, elle n'est pas seulement sourde aux arguments qui vont à son encontre, elle trie également ceux qui la confortent. Autrement dit, l'audience que trouvent les résultats d'enquêtes varie selon la nature du constat dressé. Ainsi, lorsque les résultats peuvent aller dans le sens de l'idée reçue, ici l'archétype de l'indissolubilité du lien conjugal, alors l'écho peut être considérable.

Le meilleur exemple que I'on puisse donner de ce phénomène est sans doute la publicité faite autour des résultats d'une thèse de démographie portant sur les conséquences du divorce sur la scolarité des enfants (Archambault, 2001). S'appuyant sur I'analyse quantitative de deux enquêtes portant sur les jeunes (14), cette recherche compare l'âge de fin de scolarité et le dernier diplôme obtenu par des jeunes selon la situation familiale à 18 ans, en contrôlant le milieu d'origine, mesuré par la catégorie socioprofessionnelle du père, et I'héritage scolaire et culturel, mesuré par le niveau d'étude de la mère. Le diagnostic semble clair : "Quel que soit le milieu social, la rupture du couple parental est associée à une réussite scolaire plus faible chez l'enfant. La séparation des parents avant la majorité de l'enfant réduit la durée de ses études de six mois à plus d'un an en moyenne. L'avantage scolaire lié à une enfance passée dans une famille favorisée du point de vue culturel et social semble fortement amoindri en cas de désunion familiale»(Archambault, 2002, p. 1).

L'auteur souligne également que les jeunes confrontés à la séparation et au divorce sortent plus précocement du domicile parental, de un à deux ans plus tôt, et forment des couples plus précocement (ce qui peut expliquer éventuellement la fragilité potentielle de ces couples précoces). En revanche, ils n'éprouvent pas de difficultés significativement supérieures à s'insérer professionnellement comparé aux autres membres de leur classe d'âge (surtout pour les filles). Manifestement, les conflits intergénérationnels jouent un rôle très important dans ces sorties plus précoces du ménage parental (surtout dans les cas de recomposition familiale). Á partir de l'apprécia- tion que ces jeunes font de leur trajectoire, P. Archambault montre aussi que le désir d'indépendance précoce est essentiel dans la décision d'arrêter les études. "Vouloir gagner sa vie » est le motif déterminant d'arrêt des études pour $66 \%$ de la population peu diplômée. En revanche, très peu $d^{\prime}$ entre eux font appel à leur trajectoire familiale « perturbée » pour expliquer leur parcours scolaire ( $5 \%$ en famille monoparentale et $10 \%$ en famille recomposée). Paul Archambault ne néglige pas pour autant les effets liés à la précarisation économique. Il souligne également que près de $40 \%$ de la moindre performance scolaire au bac des enfants de familles dissociées pourraient être imputables au moindre transfert économique entre générations (argent de poche, soutiens pour l'achat de livres, etc.). "Á 18 ans, les aides des parents qui se sont séparés sont moins fréquentes dans tous les milieux sociaux et, en particulier, en milieu ouvrier. La séparation des parents creuse donc l'écart social de l'inégalité des aides économiques des parents à leurs enfants lors du passage à l'âge adulte » (Archambault, 2001, p. 205).

P. Archambault se limite pratiquement au constat et ne fait qu'esquisser les mécanismes en cause (moindre contrôle scolaire exercé par les parents, persistance des conflits familiaux après la séparation, ressources économiques amoindries, effets des politiques sociales et familiales, etc.), notamment en raison de l'absence de données qualitatives approfondies susceptibles de compléter l'enquête quantitative (15). Même s'il prend certaines précautions avec les idées reçues (16), l'écho sera rapidement très important dans les médias car ces données confortent le sens commun qui veut que le divorce porte un préjudice sérieux aux enfants, avec des conséquences supposées en termes de trajectoires $d^{\prime}$ insertion socioprofessionnelle et, plus globalement, d'insertion sociale. Faudrait-il en conclure qu'il vaut toujours mieux rester en couple lorsque l'on a eu des enfants ensemble?

\section{Le conflit parental peut-être plus dévastateur que la séparation}

Une autre enquête de Thomas Piketty publiée en 2003 nuance passablement ces résultats (17). Grâce à la méthode utilisée, l'auteur met à l'épreuve les

\footnotetext{
(14) L'enquête " passage à l'âge adulte » de I'INED (1993) et la première enquête "Jeunes » complémentaire à l'enquête Emploi de l'INSEE (1992). Les données ont donc près de dix ans au moment de leur publication.

(15) À l'appui de telles données, les interprétations sont nettement plus nuancées, comme celles de Elsa Ferri, Andrew Cherlin ou Kathleen Kiernan (pour un bilan, voir Burghes, 1997).

(16) Par exemple, il prend le soin de souligner que « les difficultés scolaires des enfants de familles monoparentales semblent découler de la précarité plus que d'un effet propre, d'ordre psychologique, de la dissociation des parents » (2001, p. 162). On peut aussi regretter que P. Archambault n'ait pas mentionné les écarts considérables existant selon les pays entre ces effets de la séparation et du divorce sur la performance scolaire des enfants, du fait des politiques sociales menées dans ces pays, permettant de lutter contre ces effets négatifs et même de les annihiler complètement (Dronkers, 2003).

(17) Malgré la fréquence des interventions de ce chercheur dans la presse et les médias, il est remarquable que cette enquête (il est vrai publiée en anglais) n'ait pas connu l'écho de celle de P. Archambault.
} 
effets de sélection. II compare ainsi la performance scolaire d'enfants quelques années avant la séparation de leurs parents, puis la performance scolaire d'enfants vivant dans un ménage monoparental post-désunion. Comme il l'écrit lui-même: "Mes résultats suggèrent que les conflits parentaux (plus que la séparation elle-même) ont des effets négatifs sur les enfants et que la distribution de l'intensité des conflits au sein des couples n'a guère varié au fil du temps et qu'elle n'a pas été affectée par la réforme du divorce de 1975 » (Piketty, 2003, p. 22).

Une fois encore, ces résultats corroborent ceux de plusieurs chercheurs. On peut mentionner en particulier les travaux de Jaap Dronkers, qui figure parmi les meilleurs spécialistes de cette question des conséquences du divorce notamment sur la performance scolaire, appréciées à l'aide de recherches quantitatives et longitudinales. Dans un article de 1999 rendant compte d'une recherche menée aux Pays-Bas sur un échantillon de neuf mille enfants scolarisés au secondaire, ses conclusions sont tout à fait claires : "premièrement, le bienêtre des élèves vivant avec une mère seule est meilleur que celui d'élèves vivant dans un ménage biparental avec de forts conflits conjugaux; deuxièmement, le bien-être des élèves vivant avec une mère seule sans conflit parental et avec de fréquents contacts avec le père est moindre que celui d'élèves vivant dans une famille biparentale sans conflit entre les parents; troisièmement, le degré de conflit parental après le divorce est plus important pour le bien-être des enfants que le degré de contact avec le père "non-gardien" " (Dronkers, 1999, p. 195 ; voir aussi Dronkers et Lont, 2003 ; Dronkers et al., 2006).

Á la lumière de cet ensemble de résultats, il semble bien que la question des effets du divorce soit mal posée. Le mauvais climat relationnel dans un couple serait la variable indépendante, masquée par le fait que ces conflits peuvent en effet conduire dans de nombreux cas à une séparation ou un divorce. Mais à ne prendre en considération que cet événement visible et enregistrable, on risque de prendre une conséquence - la désunion pour la cause des difficultés éprouvées par les enfants, quelles qu'elles soient, sans chercher à remonter à la cause masquée: la mésentente des parents. Que dire dès lors, des effets du nondivorce? En effet, dans les cas où le conflit parental ne conduit pas à une séparation, il peut se révéler tout aussi, voire plus dévastateur encore que la séparation elle-même. Mais, dans ces cas, le travail sociologique est beaucoup plus délicat. Seuls les cliniciens, thérapeutes familiaux et conjugaux sont susceptibles de recevoir ces ménages en souffrance et semblent capables d'en dire quelque chose. Comment travailler sociologiquement sur les conséquences d'un non-divorce?

On comprend que ce type de situations familiales ne fasse l'objet d'aucune statistique (il est de toute évidence difficile $d^{\prime}$ en faire) et de très peu de travaux sociologiques. On peut cependant postuler que ces situations existent aujourd'hui comme hier, ce dont témoignent un certain nombre d'œuvres littéraires qui, tout en décrivant ces figures familiales du désamour, contribuaient à produire une critique de la famille bourgeoise de la fin du XIX ${ }^{\mathrm{e}}$ siècle ou de la première moitié du $X X^{\mathrm{e}}$ siècle et du conformisme de bon aloi (18). Mais en témoigne également l'importance des divorces qui interviennent aujourd'hui après le phénomène du nid vide, voire à la retraite, autrement dit au moment précisément où la question des effets du divorce sur les enfants ne se pose plus véritablement (Caradec, 1996) (19).

\section{Les familles du désamour}

Ces familles où le désamour s'est cristallisé, conduisant à un renoncement total ou partiel à la sexualité (on fait chambre à part, on ne dort plus dans le même lit, souvent au vu et au su de ses propres enfants, voire du reste de sa famille et parfois même de ses amis), demeurent en grande partie invisibles sociologiquement (20). Si l'existence de telles situations, où s'entremêlent amertume et parfois même de la haine, semble pouvoir caractériser la fin du XIX ${ }^{\mathrm{e}}$ siècle et la bourgeoisie du début du $X X^{\mathrm{e}}$ siècle dans une sorte de syndrome Bovarien, elles paraissent aujourd'hui hautement improbables. Rien ne semble pouvoir justifier de s'installer dans un tel désamour, sauf peut-être lorsque I'on conçoit que ces couples sont tout au plus dans "l'avant-divorce ». Mais que dire de tels cas lorsque cette situation se prolonge durant des années, voire des décennies? Ne peut-on parler alors d'un modèle de ménage où l'on vit ensemble séparés, «living together apart»?

\footnotetext{
(18) On peut mentionner les œuvres suivantes qui sont particulièrement exemplaires de cette thématique: Le nœud de vipères de François Mauriac, Le destin de Mr Crump de Ludwig Lewisohn, romans des années 1930 et Chroniques maritales de Marcel Jouhandeau, notamment.

(19) À titre indicatif, près de 15000 femmes et hommes ont divorcé en 2005 sur les 152000 divorces prononcés en Métropole cette même année soit près de $15 \%$.

(20) La dernière enquête Contexte de la sexualité en France, menée en 2006 par l'INED et l'INSERM, fournit quelques premières pistes sur les « chambres à part », sans bien sûr que cela témoigne d'une quelconque mésentente. Ainsi, dans cette enquête, 2,5\% des hommes et des femmes disent dormir dans des chambres différentes, et ce d'autant plus qu'ils sont plus âgés (respectivement $6 \%$ des 50-59 ans et $6 \%$ des 60-64 ans). Je remercie Michel Bozon de m’avoir fourni ces chiffres.
} 
Ces couples ne sont-ils tout au plus que des traces du passé, d'un passé qu'ont connu nos aïeux, mais qui n'aurait plus aujourd'hui de raison d'être puisque le divorce est possible et relativement banalisé ? Ne peut-on au contraire imaginer que la crainte des conséquences de la séparation conduit à attendre, reporter, s'adapter, voire renoncer? Mais on peut aussi faire l'hypothèse que la décision de la séparation n'est jamais dénuée d'ambivalence en raison de la dépendance que l'on a construite au fil du temps, des routines conjugales qui constituent tout à la fois un confort et une prison.

Les Chroniques maritales de Marcel Jouhandeau sont très éclairantes de ces phénomènes de dépendance mutuelle et d'ambivalence. Élise dit ainsi : "Pour une vraie femme, il n'y a de jeu préférable à celui de retenir un homme auprès d'elle, malgré lui et d'autant plus étroitement qu'il le souhaite moins. Dans un couple souvent celui qu'on croit le moins fort ne demeure que par pitié auprès de l'autre, géant dont il est le seul à connaître la faiblesse. C'est ainsi que ma solitude est doublée par la présence d'un être que j'ai enchaîné à moi pour me garder: tentation merveilleuse que d'élever toujours plus haut les murs de sa prison pour s'empêcher de les franchir à force de danger et de désirer d'autant plus de les franchir qu'ils sont plus hauts» (p. 163)... Ou bien encore: "Ce n'est peut-être pas elle que tu aimes, mais sa présence et c'est bien plus fort que si tu l'aimais. II est des êtres en effet qu'on aime et l'on ne peut pas demeurer avec eux longtemps. II en est d'autres au contraire qu'on croit détester, mais la vie ne semble possible que si on les entend bouger dans l'ombre et dès qu'ils s'éloignent, on est perdu " (p. 165)... Et le narrateur (M. Jouhandeau) d'ajouter : "Même si tu la quittais, tu penserais à elle et d'autres servitudes plus graves t'empêcheraient de penser librement. Même si tu la quittais, tu ne serais pas libre... Sans doute si je ne suis sensible chez une femme à rien plus qu'à la délicatesse des attentions, j'ai souffert de n'en trouver pour ainsi dire aucune chez ma propre femme, mais parce qu'elle est ainsi, c'est par là qu'elle m'intéresse à elle et aussi à ma propre histoire» (Jouhandeau, 1962).

Interviewé par Vincent Caradec lors de sa thèse, monsieur Berg évoque très clairement ce confort de I'habitude, cette ambivalence sans amour, ce qui le conduit à commenter son hésitation à quitter sa femme: "On sait l'autre. On le connaît parfaitement. On sait tout de lui, à tel point que la vie est aussi plus confortable dans le sens où il n'est pas besoin de dire par exemple: "Non, je n'aime pas les pommes de terre" ou "je ne mange jamais de frites". Je donne un exemple tout à fait banal. Y'a une espèce de respiration à la fois compliquée par le fait qu'on ne s'entend plus, mais rendue facile par rapport à une autre coexistence avec une autre, où tout serait à réapprendre, à refaire et à reconjuguer. C'est paradoxal, ce que je dis, mais c'est deux entités qui se conjuguent quelque part. Le fait qu'on connaît tellement l'autre qu'on ne l'aime plus et qu'on le connaît aussi tellement qu'il nous facilite la vie parce qu'il sait comment naviguer avec nous" (Caradec, 1996, p. 256-257).

\section{Vivre ensemble séparés}

Ce processus de désamour, qui peut conduire à un véritable mode de vie, ensemble et séparés, peut être long et produire cet étrange paradoxe conjugal. monsieur Jeantot, également interviewé dans le cadre de la recherche de V. Caradec le raconte en ces termes: " I/ y avait déjà cinqsix ans qu'on avait fait chambre à part, parce que comme j'aimais bien lire au lit le soir avant de m'endormir, c'était toujours: "Ah tu lis, je ne peux pas $\mathrm{m}^{\prime}$ endormir ». Elle ne dormait jamais. Le matin, c'était : "Tu fais du bruit, tu me réveilles : le matin, c'est là où je dors ». Un jour, je lui dis : "Écoute, on a trois chambres, tu en prends une, moi j'en prends une, comme ça je ne te réveillerai pas le matin et, le soir, je pourrai lire »... Nos relations sexuelles se sont estompées depuis un an. Là, c'était pratiquement tombé à zéro, elle n'avait plus envie de faire l'amour, elle n'avait plus envie de moi. Moi, je n'en avais plus tellement envie non plus. Ce qui fait que, depuis un an, c'était vraiment le calme plat »(Caradec, op.cit., p. 254).

On peut illustrer cette même "logique » dans le récit d'un cas de "living together apart» (21). Voici ce que dit Jacques, âgé de 54 ans, encore marié, père de trois enfants de 20, 18 et 11 ans, et qui s'est "installé » dans une relation de ce type depuis près de dix ans : "Notre histoire a toujours été tumultueuse. Au départ, c'était d'abord une passion, parfois féroce, qui nous a liés, ou devrai-je dire qui nous a fait fusionner... Mais, à force d'à-coups, de crises, de conflits, d'engueulades, cette fusion qui exigeait trop, qui exigeait tout, s'est transformée en douleur et parfois en violence. Que de blessures accumulées pour en arriver à notre situation... Aujourd'hui, l'idée de la toucher est devenue un danger. La sexualité qui nous a tant donné est devenue un danger, celui de la réconciliation, alors que justement notre expérience nous a appris que ces réconciliations sur l'oreiller ne

(21) Nous menons depuis un an une recherche exploratoire sur ces situations conjugales parallèlement à nos travaux sur les questions de conciliation vie familiale-vie professionnelle. 
changent rien quant au fond. Nous ne sommes pas faits l'un pour l'autre».

Dans son récit, Jacques souligne l'ambivalence et explique comment elle les a conduit à rester ensemble séparés: "Il ne fait aucun doute que nous sommes malheureux ensemble. Nous faisons chambre à part depuis pratiquement dix ans et lorsque les circonstances nous y obligent, nous prenons garde de ne pas nous effleurer. C'est I'hôtel du cul tourné... Et pourtant nous quitter est difficile pour mille raisons. Les enfants, tout d'abord. Nous aurions dû le faire il y a plus de dix ans, avant la naissance du dernier. Aujourd'hui, on a un peu l'impression qu'il faut attendre que les enfants soient grands, indépendants. On craint que cela soit encore pire séparés... Il y a aussi la peur de l'appauvrissement. Le divorce est un grand saut dans la précarité... Mais parfois, je pense que l'essentiel n'est pas là. Nous restons ensemble, car nous avons par habitude construit cette dépendance ».

V. Caradec avait déjà repéré ces phénomènes dans les récits de personnes retraitées confrontées à l'idée de la rupture. Ainsi une lectrice de Notre temps dont il restitue le témoignage écrit ceci : "J'ai 60 ans et je viens de prendre ma retraite. Mon mari, avec lequel je ne me suis jamais bien entendue, est déjà retraité depuis deux ans. Toute ma vie, j'ai tenu le coup à cause des enfants. Ils nous ont quittés maintenant pour vivre leur vie et je suis complètement désemparée. Mon mari est tyrannique et grossier, brutal parfois. II fait fuir tous nos amis, et je me vois mal le supportant toute la journée. D'un autre côté, une séparation à nos âges pose tellement de problèmes! Je crains aussi ses réactions si je lui parle d'une telle éventualité... À votre avis, qu'est-ce que je peux faire?»;

Á la différence du XIXe siècle décrit dans Madame Bovary, la pression sociale, le respect des normes bourgeoises pèsent beaucoup moins que la question de l'enfant et de sa socialisation. La nouvelle norme contemporaine: «rester parent si l'on ne peut plus être un couple», peut prendre non seulement la figure de la séparation et de la circulation des enfants, mais aussi celle du living together apart. Bien sûr, cette cohabitation souffrante suppose, pour éviter les effets destructeurs, de respecter un certain nombre de règles pour épargner le plus possible les enfants du climat de désamour, tout comme pour les couples séparés physiquement. Mais ces règles sont très difficiles à tenir dans la cohabitation, comme le soulignent les deux cas suivants. Dans la famille Berg, interviewée par V. Caradec: "On a des enfants à qui on essaie de donner une espèce d'image de leurs parents qui ne soit trop négative... La grande question est de savoir si on ne donne pas une image plus négative en restant dans la mésentente, vis-à-vis d'eux, que si on partait, mais comme on a l'impression d'être quelque part utile, malgré nos chamailleries, auxquelles ils se sont accommodés plus ou moins, donc on souhaite les mener le plus loin possible»(Caradec, 1996, p. 252).

Mais c'est surtout l'histoire de Roland qui éclaire le mieux cet impact tant redouté de ce mode de vie. Après vingt ans de vie de couple et près de sept ans d'enfer conjugal, qui ont débouché sur un mode de vie living together apart, il émet le diagnostic suivant: "Notre vie quotidienne s'est profondément dégradée. Je ne pouvais plus la supporter et elle non plus. Nous avons été si loin dans cette détestation quotidienne que j'ai souvent voulu partir. Certaines scènes m'ont conduit à partir une nuit à l'hôtel, voire quelques jours, à plusieurs reprises, car dans notre guerre conjugale, on en arrivait à oublier les enfants et à nous insulter à côté d'eux... Mais en fait je craignais de me trouver éloigné des enfants. Elle m'a souvent menacé de partir avec eux, loin, et de me faire une guerre à distance dans le cadre d'un divorce hyperconflictuel... Le genre "j'aurai ta peau ». Alors, j'ai choisi d'attendre, de rester aux côtés de mes enfants et de les voir grandir... Maintenant, je mesure que ce choix est aussi une faiblesse. Ils ont bien grandi à mes côtés et moi à leur côté, mais au milieu d'un véritable chaos conjugal dont je commence seulement à mesurer les conséquences. Que de scènes, de coups de gueule dont ils auraient été dispensés, si nous avions été assez courageux pour rompre... Aujourd'hui, je consulte un psychologue et mes deux filles aussi. Il est clair qu'elles ont souffert et qu'elles sont profondément marquées par ce contexte familial merdique... Nous avons quelque chose à réparer et je suis sûr qu'elles nous en veulent d'avoir été si lâches et égoïstes ».

L'exemple de ces ménages peut donc permettre de forger de nouvelles hypothèses sur l'importance de la norme du lien parental qui survit au lien conjugal. En effet, dans le discours de quelques ménages de ce type, cette question de la parentalité comme justification de ce choix de vie est particulièrement centrale. II pourrait y avoir un "prix» élevé lié au fait de se considérer d'abord comme des parents responsables du bien-être de ses enfants, et sommés de rester ensemble, pour eux. Preuve, s'il en était besoin, que la question de la norme et des prescriptions sociales concernant la vie privée et le rôle de parent est particulièrement prégnante aujourd'hui (Commaille et Martin, 1998), mais aussi que l'archétype de I'indissolubilité évoqué par Jean Carbonnier n'a pas dit son dernier mot. 


\begin{tabular}{l|l} 
& \\
Références & bibliographiques
\end{tabular}

Abel O. et S. Laugier, 2005, Le divorce : une rupture démocratique, Postface à la réédition de John Milton, Doctrine et discipline du divorce (1644), traduction C. Tournu, Paris, Belin.

Amato Paul R., 1994, Life-span adjustment of children to their parents' divorce, The Future of Children, vol. $4, \mathrm{n}^{\circ} 1$.

Archambault P., 2002, Séparation et divorce : quelles conséquences sur la réussite scolaire des enfants? Population et sociétés, INED, $\mathrm{n}^{\circ} 379$.

Archambault P., 2001, "Le devenir des enfants de familles dissociées. Approche sociodémographique à partir des enquêtes sur la jeunesse en France », thèse pour le doctorat de sociologie, Université de Paris-5 René-Descartes.

Archambault P., 1998, Les difficultés d'accès au diplôme des enfants de familles dissociées, Espace, population, société, $\mathrm{n}^{\circ} 2$.

Aubusson de Cavarlay B., 2002, Les chiffres de la délinquance : production et interprétation, in Etat, société et délinquance (sous la dir. de P. Tronquoy), Paris, Cahiers français, $n^{\circ} 308$.

Burghes L., 1997, Lone parenthood and family disruption, Londres, Family Policy Studies Centre.

Caradec V., 1996, Le couple à l'heure de la retraite, Rennes, Presses universitaires de Rennes.

Carbonnier J., 1995, Essai sur les lois, Répertoire du notariat Defrénois, $2^{e}$ édition.

Carbonnier J., 1988, Flexible droit. Pour une sociologie du droit sans rigueur, Paris, LGDJ, 2001.

Cavell S., 1993, À la recherche du bonheur. Hollywood et la comédie du remariage (1981), Traduction C. Fournier et S. Laugier, Paris, Cahiers du Cinéma.

Chambaz C. et Martin C., 2001, Lone parents, employment and social policy in France: lessons from a familyfriendly policy, in Lone parents, employment and social policy. Cross-national comparisons (sous la dir. de Millar J. et Rowlingson K.), Bristol, Policy Press:129-150.

Cherlin A.-J., 1999, Public and Private Families. An introduction, McGraw-Hill College (2e édition).

Comité de pédiatrie psychosociale, 2000, La promotion de la santé mentale pour les enfants de parents qui se séparent, Paediatrics et Child Health, 5(4):237-240.

Commaille J. et Martin C., 1998, Les enjeux politiques de la famille, Paris, Bayard.

Debardieux E., 2002, La violence en milieu scolaire : statistiques officielles, victimisations et multivictimisations, in État, société et délinquance, (sous la dir. de. Tronquoy P.), Paris, Cahiers français n 308:50-58.

Dronkers J., Kalmijn M. et Wagner M., 2006, Causes and consequences of divorce: cross-national and cohort differences, European Sociological Review, vol. 22, n 5. Dronkers J., 2003, Family policies and children's school achievement in single - versus two-parent families, Journal of Marriage and Family, $\mathrm{n}^{\circ}$ 65:681-699.

Dronkers J., 1999, The effects of parental conflicts and divorce on the well-being of pupils in dutch secondary education, European Sociological Review, vol. 15, n²:195-212.

Heuyer G., 1952, Introduction à la psychiatrie infantile, Paris, PUF, coll. "Sup » (nouvelle édition 1966).

Dronkers J. et Lont A., 2003, Changes in the relation between parental divorce and the well-being of their adolescent children during the 1984-1999 period in the Netherlands, European University Institute Working Papers, SPS n²003/12.

Jouhandeau M., 1962, Chroniques maritales, précédé de Élise, Paris, Gallimard (NRF).

Lefaucheur, N., 1996, Dissociation familiale et délinquance juvénile ou la trompeuse éloquence des chiffres, in Familles et politiques sociales (sous la dir. de Le Gall D. et Martin C.), Paris, L'Harmattan. 
Lefaucheur N., 1989, "Dissociation familiale et délinquance juvénile : les avatars scientifiques d'une représentation sociale », rapport de recherche pour la CNAF.

Le Gall D. et Martin C., 1988, "Le réseau de parenté après la désunion », rapport de recherche pour la Caisse nationale des allocations familiales, Centre de recherche sur le travail social, Université de Caen.

Lewis J., 1997, Lone mothers in european welfare regimes. Shifting policy logics, London, Jessica Kingsley Publishers.

Lewisohn L., 1996, Le destin de Mr Crump, Paris, Phébus (1're édition 1947).

Martin C., 2006, Divorce, in Le dictionnaire des sciences humaines (sous la dir. de Mesure S. et Savidan P.), Paris, PUF:290-293.

Martin C., 2003 a, "La parentalité en question. Perspectives sociologiques », rapport remis au Haut conseil de la population et de la famille, publié sur le site de La Documentation française.

Martin C., 2003 b, Les savoirs aux prises avec l'opinion : I'exemple des effets du divorce, Lien social et politiques, $\mathrm{n}^{\circ}$ 50:57-71.

Martin C., 1997, L'après-divorce. Lien familial et vulnérabilité, Rennes, Presses universitaires de Rennes.

Mauriac F., 1933, Le nœud de vipères, Paris, Le livre de poche.

Milton, J., 2005, Doctrine et discipline du divorce (1 ${ }^{\text {re }}$ édition 1644), traduction C. Tournu, Paris, Belin.

Ministère de la Justice Canada, 1997, "Les effets du divorce sur les enfants. Analyse documentaire ", document de travail, Division de la recherche et de la statistique.

Mucchielli L., 2000 a, «Familles et délinquances. Un bilan pluridisciplinaire des recherches francophones et anglophones ", rapport de recherche pour la Caisse nationale des allocations familiales, CESDIP. Études et données pénales.

Mucchielli L., 2000 b, La dissociation familiale favorise-t-elle la délinquance ? Arguments pour une réfutation empirique, Recherches et Prévisions, CNAF, n 61:35-50.

Mucchielli L., 2000 c, Le contrôle parental du risque de délinquance juvénile : un bilan des recherches, Les Cahiers de la sécurité intérieure, $\mathrm{n}^{\circ}$ 42:127-146.

Mucchielli L., 2001, Violences et insécurité. Fantasmes et réalités dans le débat français, Paris, La Découverte.

Piketty T., 2003, "The impact of divorce on school performance: evidence from France, 1968-2002», Discussion Paper Seies, Centre for Economic Policy Research: $n^{\circ} 4146$ (www.cepr.org/pubs/dps/DP4146.asp).

Roché S., 2000, Les facteurs de la délinquance des jeunes. Analyse à partir d'une enquête de délinquance auto-déclarée, Les Cahiers de la sécurité intérieure, $\mathrm{n}^{\circ}$ 42:37-41 voir aussi http://web.upmfgrenoble.fr/cerat/Recherche/pagesperso/Roche.html).

Théry I. (dir.), 2004, Incompatibilité d'humeur. Du droit au divorce à la théorie du mariage chez John Milton, Esprit, juillet:71-94.

Tournier V., 2003, Le rôle de la famille dans la délinquance, in En quête de sécurité. Causes de la délinquance et nouvelles réponses (sous la dir. de Roché S.), Paris, Armand Colin:101-114.

Vanderschelden M., 2006, Les ruptures d'union : plus fréquentes mais pas plus précoces, INSEE Première, $n^{\circ} 1107$. 
